\title{
Muscle differentiation induced up-regulation of calcium-related gene expression in quail myoblasts
}

\author{
Jeong-Woong Park', Jeong Hyo Lee', Seo Woo Kim², Ji Seon Han², Kyung Soo Kang ${ }^{3}$, \\ Sung-Jo Kim ${ }^{4, *}$, and Tae Sub Park ${ }^{1,2, *}$
}

\author{
* Corresponding Authors: \\ Sung-Jo Kim \\ Tel: +82-41-540-5571, Fax: +82-41-540-9538, \\ E-mail: sungjo@hoseo.edu \\ Tae Sub Park \\ Tel: +82-33-339-5721, Fax: +82-33-339-5763, \\ E-mail: taesubpark@snu.ac.kr \\ ${ }^{1}$ Institute of Green-Bio Science and Technology, Seoul \\ National University, Pyeongchang 25354, Korea \\ ${ }^{2}$ Graduate School of International Agricultural \\ Technology, Seoul National University, Pyeongchang \\ 25354, Korea \\ ${ }^{3}$ Bio Division, Medikinetics, Inc., Pyeongtaek 17792, \\ Korea \\ ${ }^{4}$ Division of Cosmetics and Biotechnology, Hoseo \\ University, Asan 31499, Korea \\ ORCID \\ Sung-Jo Kim \\ https://orcid.org/0000-0003-4590-3644 \\ Tae Sub Park \\ https://orcid.org/0000-0002-0372-5467
}

Submitted Apr 16, 2018; Revised May 9, 2018; Accepted May 29, 2018
Objective: In the poultry industry, the most important economic traits are meat quality and carcass yield. Thus, many studies were conducted to investigate the regulatory pathways during muscle differentiation. To gain insight of muscle differentiation mechanism during growth period, we identified and validated calcium-related genes which were highly expressed during muscle differentiation through mRNA sequencing analysis.

Methods: We conducted next-generation-sequencing (NGS) analysis of mRNA from undifferentiated QM7 cells and differentiated QM7 cells (day 1 to day 3 of differentiation periods). Subsequently, we obtained calcium related genes related to muscle differentiation process and examined the expression patterns by quantitative reverse-transcription polymerase chain reaction (qRT-PCR).

Results: Through RNA sequencing analysis, we found that the transcription levels of six genes (troponin C1, slow skeletal and cardiac type [TNNC1], myosin light chain 1 [MYL1], MYL3, phospholamban $[P L N]$, caveolin $3[C A V 3]$, and calsequestrin 2 [CASQ2]) particularly related to calcium regulation were gradually increased according to days of myotube differentiation. Subsequently, we validated the expression patterns of calcium-related genes in quail myoblasts. These results indicated that TNNC1, MYL1, MYL3, PLN, CAV3, CASQ2 responded to differentiation and growth performance in quail muscle.

Conclusion: These results indicated that calcium regulation might play a critical role in muscle differentiation. Thus, these findings suggest that further studies would be warranted to investigate the role of calcium ion in muscle differentiation and could provide a useful biomarker for muscle differentiation and growth.

Keywords: Quail; Muscle Differentiation; Calcium; RNA-sequencing

\section{INTRODUCTION}

The most important economic traits in poultry industry are egg production and amount of meat. Thus, genetic selection and breeding programs have made efforts to improve such quantitative traits [1]. Particularly, chicken meat yield is closely related to skeletal muscle growth and so, the understanding of muscle proliferation and differentiation could provide the critical tool for improving the meat amount.

Muscle growth and differentiation in vertebrates including Aves are controlled by a complicated processes. For in vitro analysis of muscle growth, the cell culture systems of muscleoriginated cells have been established in various species [2]. Many studies on myoblasts which are the precursors of myotubes were conducted to examine the regulatory pathways for muscle differentiation [3]. In Aves, QM7 cell line derived from quail myoblast has been utilized for studying the myogenesis mechanisms [4,5]. In mammals, the onsets of myopathy were caused by the dysregulated myogenic factors such as myogenic regulatory factors 
(MRFs) [6]. MRFs include a group of four proteins; $M y o D$, Myf5, Myogenin, and MRF4 [7,8]. Those MRFs regulate cell cycle arrest of precursor muscle cells and regenerative proliferation. Additionally, MRFs induce muscle differentiation and sarcomere assembly process by activating sarcomere and expressing muscle specific genes [8].

Calcium ion is well known to have various biological functions in body as formation of bone structure, wound healing, and hormone seduction [9]. In muscle, calcium ion acts as a regulatory molecule and signaling molecule in muscle fiber [10]. Generally, calcium signal in muscle is closely related to muscle contraction and muscle relaxation [11]. The muscle contraction pathways regulated by calcium ions show three major mechanisms [11-13]; i) Troponin-tropomyosin complex system which is related to actin-filament in skeletal muscle and cardiac muscle, ii) Myosin light chain kinase mechanism which occurs during muscle contraction with calmodulin in vertebrate's smooth muscles, iii) Calcium ions bind directly to myosin and induce muscle contraction. Thus, calcium ions are very important for muscle contraction and relaxation but the roles of calcium ions during muscle differentiation have not yet been studied.

Based on mRNA sequencing analysis of quail myoblasts (QM7) during 3 days of myotube differentiation, we analyzed the differentially expressed genes (DEGs) and validated the expression profiles of calcium-related genes to investigate calcium regulatory processes during myotube differentiation.

\section{MATERIALS AND METHODS}

\section{Quail myoblast culture}

According to the previous reports [4,5], QM7 cells (American Type Culture Collection, Manassas, VA, USA) were maintained at $37^{\circ} \mathrm{C}$ in an atmosphere of $5 \% \mathrm{CO}_{2}$ and $60 \%$ to $70 \%$ relative humidity with Medium 199 containing 10\% fetal bovine serum (FBS; Invitrogen, Carlsbad, CA, USA), 2\% chicken serum (Sigma- Aldrich, St. Louis, MO, USA), and $1 \times$ antibioticantimycotic (Invitrogen, USA) by subculturing the cells at $70 \%$ confluency. To induce myotube differentiation at $90 \%$ confluency, the differentiation medium containing $0.5 \%$ FBS and $1 \times$ antibiotic-antimycotic was changed and half of the medium was replaced daily with fresh differentiation medium.

\section{Assembly of RNA-sequencing data of quail transcripts} Total mRNAs were isolated from the differentiated QM7 cells during 3 days of myotube differentiation as well as the undifferentiated QM7 cells, and all of samples were triplicated. We generated RNA-sequence data in the QM7 cells. Sequencing of an RNA-sequencing library for each sample was carried out using Illumina HiSeq2500 (Illumina, San Diego, CA, USA) in order to generate 100 pair-end reads. These sequences were aligned and mapped against the chicken reference genome using TopHat for paired-end sequences.

\section{Total RNA isolation cDNA synthesis}

To validate the expression patterns of calcium-related genes, total mRNAs were isolated from the undifferentiated QM7 cells and differentiated QM7 cells (day 1 to day 3 of differentiation periods). The harvested cells were dissolved using 400 $\mu \mathrm{L}$ of TRIzol (Invitrogen, USA) and $100 \mu \mathrm{L}$ of chloroform was added to remove the organic solvent. After final washing with 75\% ethanol, mRNA pellets were dissolved in RNase-free water. The extracted mRNAs were confirmed by measuring the absorbance at $230 \mathrm{~nm}$ and $260 \mathrm{~nm}$ using a spectrophotometer (NanoDrop 2000, Thermo Scientific, DE, USA) and stored at $-70^{\circ} \mathrm{C}$ for the next experiments.

To synthesize cDNA, $2 \mu \mathrm{g}$ of RNA, $1 \mu \mathrm{L}$ of oligo-dT (Invitrogen, USA), and $1 \mu \mathrm{L}$ of RNase-free water were added and then the mixture was denatured at $80^{\circ} \mathrm{C}$ for $3 \mathrm{~min}$. Subsequently, the cDNA was synthesized using $4 \mu \mathrm{L}$ of $5 \times \mathrm{RT}$ buffer, $5 \mu \mathrm{L}$ of $2 \mathrm{mM}$ deoxynucleotide (dNTP), $0.5 \mu \mathrm{L}$ of RNase inhibitor (Promega Corporation, Madison, WI, USA) and $1 \mu \mathrm{L}$ of Moloney Murine Leukemia Virus reverse transcriptase (Promega, USA).

\section{Polymerase chain reaction and quantitative polymerase chain reaction amplification}

The polymerase chain reaction (PCR) reactions to amplify the target genes in the CDNA were carried out under the following conditions; $1.8 \mu \mathrm{L}$ dNTP, $2 \mu \mathrm{L} 10 \times$ buffer, $0.2 \mu \mathrm{L}$ Taq, and $12 \mu \mathrm{L}$ distilled water were added to $2 \mu \mathrm{L}$ of $50 \mathrm{ng} / \mu \mathrm{L}$ diluted $\mathrm{cDNA}$, and $5 \mathrm{pmol} / \mu \mathrm{L}$ diluted forward primer and reverse primer. The PCR was carried out in a total volume of $20 \mu \mathrm{L}$ and the $\mathrm{PCR}$ procedure was; denaturation at $94^{\circ} \mathrm{C}$ for $10 \mathrm{~min}$, and a second denaturation at $94^{\circ} \mathrm{C}$ for $30 \mathrm{~s}$, followed by annealing at $55^{\circ} \mathrm{C}$ for $30 \mathrm{~s}$ and extension at $72^{\circ} \mathrm{C}$ for $30 \mathrm{~s}$. This step was repeated for 38 cycles and then a final extension was performed at $72^{\circ} \mathrm{C}$ for $10 \mathrm{~min}$. The band was confirmed on UV light using a 1.5\% SeaKem LE agarose gel (Lonza, Rockland, MD, USA). For quantification of calcium-related genes, quantitative reverse transcription-PCR (qRT-PCR) analysis was performed using the iCycler iQ Real-time PCR detection system (Bio-Rad, Hercules, CA, USA) and EvaGreen (Biotium, Fremont, CA, USA). qRT-PCR data of the target genes were normalized relative to beta-actin gene expression and calculated using the $2^{-\Delta \Delta \mathrm{Ct}}$ method.

\section{Bioinformatic analysis}

To identify enriched gene ontology (GO) terms, we used DAVID web based bioinformatics tool. A GO enrichment test was performed with a cut-off; raw p value $>2.00 \mathrm{E}$, false discovery rate $(\mathrm{FDR})>2.5 \mathrm{E}$. In addition, the calcium specific functional cluster was conducted with DAVID. Heat-map visualizations were performed with web-based bioinformatics 
toll shinyheatmap tool. The amino acid sequences of candidate gene in various species were obtained Ensembl 62. Amino acids were aligned using Multiple Sequence Comparison by Log-Expectation (MUSCLE) (http://www.ebi.ac.uk/ Tools/ msa/muscle/).

\section{Statistical analysis}

Statistical analysis was performed using Student t-tests in the SAS software (ver. 9.3; SAS Institute, Cary, NC, USA). Significant differences among the different groups were analyzed using the general linear model in SAS. Differences among treatments were deemed to be significant when $\mathrm{p}<0.05$.

\section{RESULTS}

\section{Identification of differentially expressed genes during myotube differentiation}

When QM7 cells were differentiated under the differentiation conditions, QM7 cells were gradually differentiated to myotubes during 3 days (Figure 1A). In addition, the areas of the differentiated myotubes constantly increased according to days of myotube differentiation (Figure 1B). To investigate the muscle differentiation-related genes, we conducted highthroughput RNA sequencing analysis for the undifferentiated QM7 cells and differentiated QM7 cells (day 1 to day 3 of differentiation periods). Based on the sequencing data, we aligned
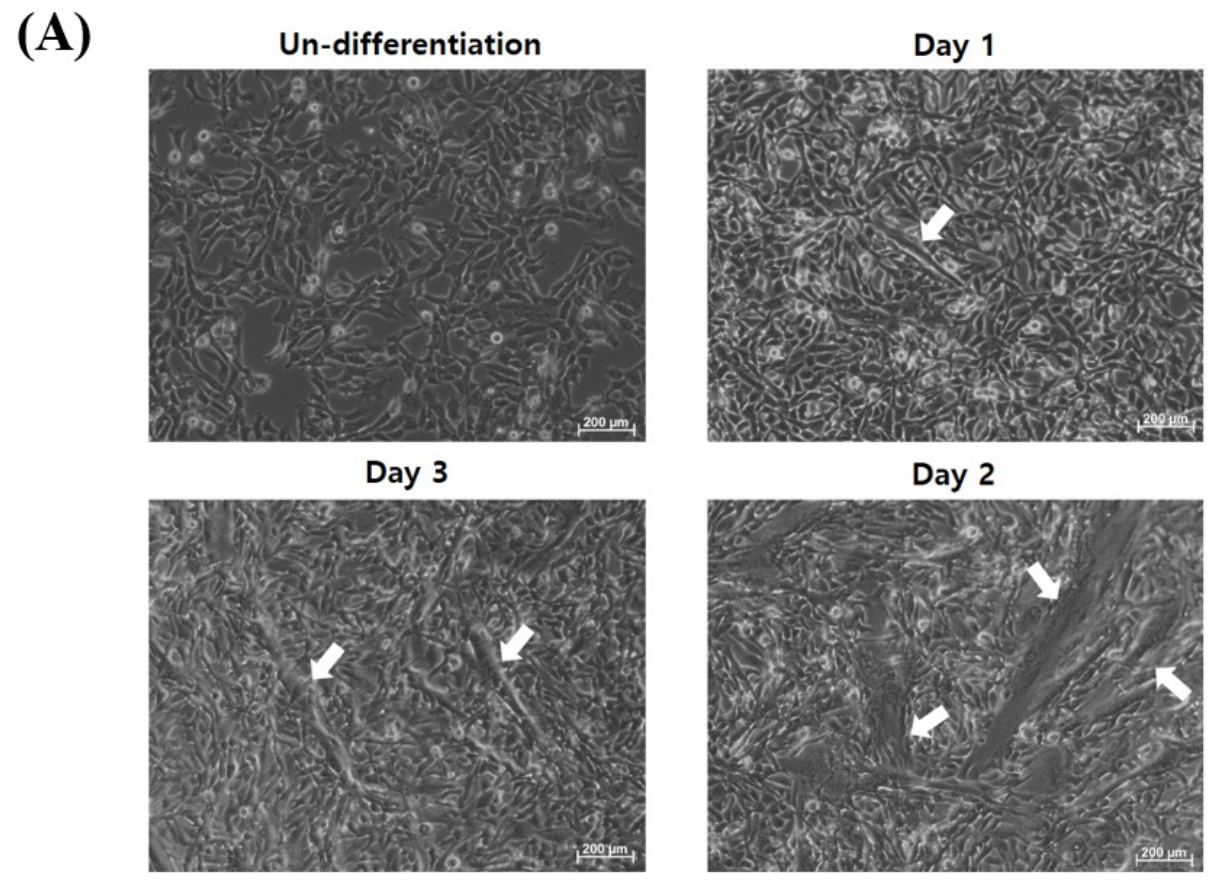

(B)

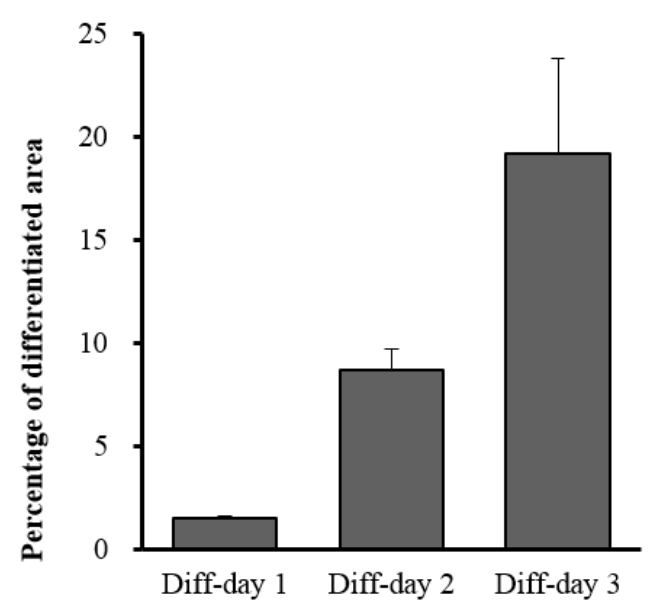

Figure 1. Myotube differentiation of QM7 myoblasts. (A) Morphology of QM7 cells during differentiation periods. QM7 cells transformed into myotubes (arrows) during differentiation. (B) Comparison of differentiated areas during differentiation periods. Percentages of differentiation areas were measured by calculating the occupied myotube area in total areas during myotube differentiation. 
and sorted-out 6,687 genes. Among the expressed transcripts, we calculated the expression levels of all genes from each undifferentiated or differentiated sample (day 1 to day 3 of differentiation periods). In Venn diagram of the comparative analysis, we identified 151 DEGs (cutoff; fold change $>2$, average of normalized read counts $(\log 2)>2$, p value $<0.01$ ) including 123 up- and 28 down-regulated genes (Figure 2A). We also conducted the comparative analysis of DEGs between day 1 to day 3 of differentiation periods and show the Venn diagram in Figure 2B. By comparing with the undifferentiated QM7, 37 and 36 transcripts were identified as up- and down-regulated genes, respectively after day 1 of myotube differentiation. In day 2 of differentiation, 29 and 15 genes were up- and down-regulated. Lastly, 86 and 47 were up- and down-regulated in day 2 of differentiation.
Functional annotation and selection of calcium-related differentially expressed genes

Based on mRNA sequencing data, we clustered muscle differentiation-related genes and summarized biological process gene ontology of DEGs in QM7 cells after myotube differentiation (Table 1). The most significant enriched terms were 'skeletal muscle contraction', 'negative regulation of calcium ion transmembrane transporter activity' and 'branch elongation of an epithelium' (Fold enrichment $=15.09$, $\mathrm{p}$ value $=$ 2.17E-03, FDR $=4.93 \mathrm{E}-02$ ). In the result of biological process GO of DEGs, the calcium ion-related process was one of the significant enriched terms. To investigate calcium ion functions during muscle differentiation, we sorted calcium-related genes based on biological process GO (Table 2). Calciumspecific biological process $\mathrm{GO}$ analysis was conducted and 9 calcium-related specific terms were summarized for mus-

(A)

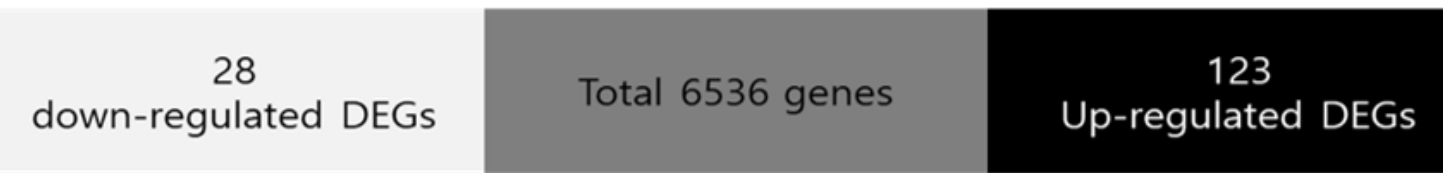

(B)

Down regulated gene

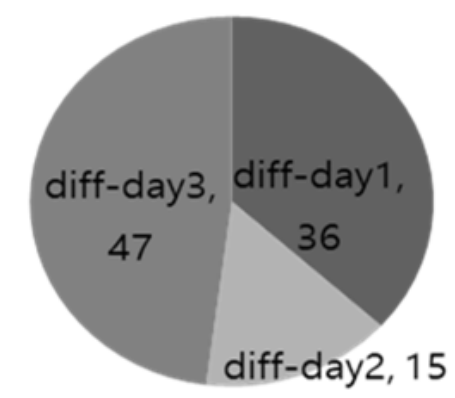

up regulated gene

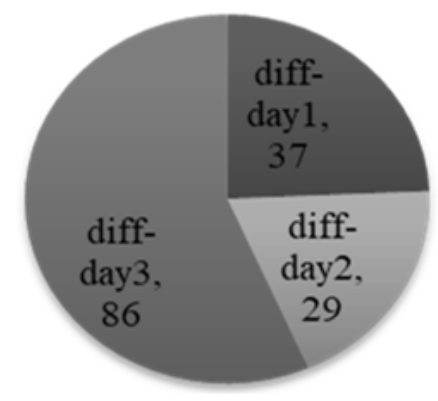

(C)

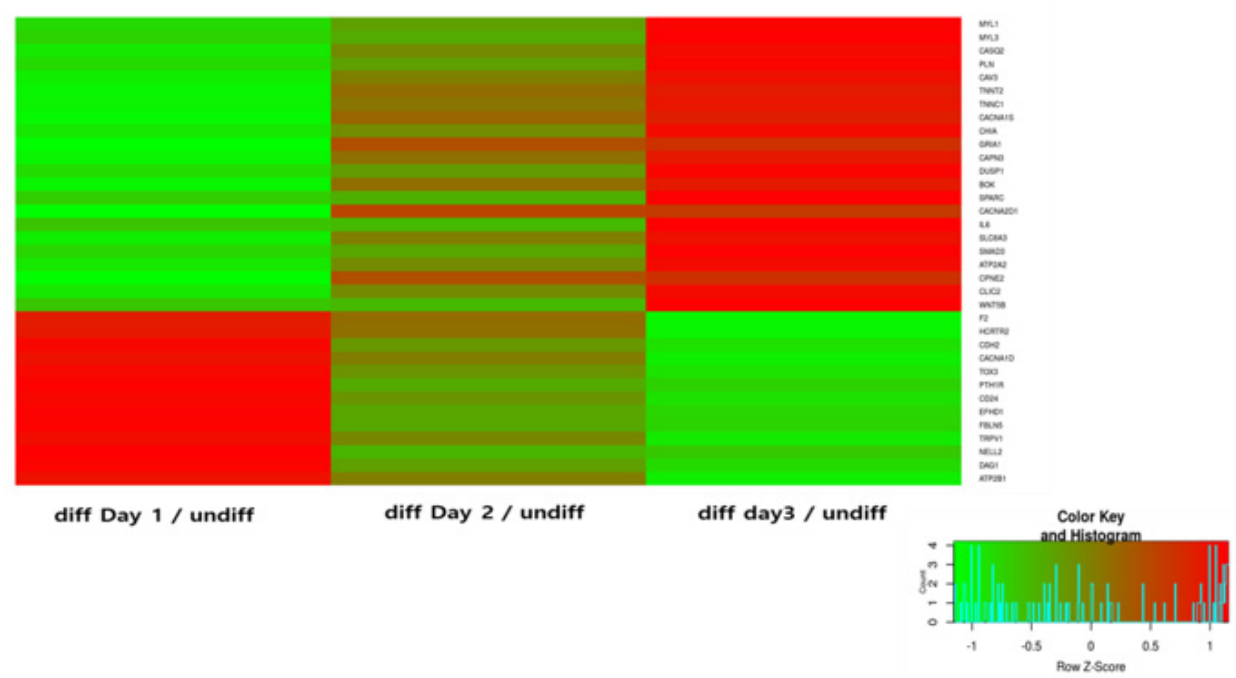

Figure 2. The Venn diagrams and heat map for differentially expressed genes (DEGs) during myotube differentiation. (A) Enhanced DEGs during myotube differentiation in QM7 cells (white bars, >2-fold down-regulated genes; black bars, >2-fold up-regulated genes; gray bars, not differentially expressed). (B) The three pie charts displayed the compositions of the DEGs during myotube differentiation periods (day 1 to day 3) compared to the undifferentiated QM7 cells. (C) Heatmap analysis of calcium-related DEGs during myotube differentiation periods (day 1 to day 3) compared to the undifferentiated QM7 cells. Color from green to red indicates low to high expression. 
Table 1. Biological process gene ontology (GO) analysis of differentially expressed genes (DEGs) which were specifically up-regulated in myotube differentiation QM7 cells (cutoff; raw $p$ value $>2.00 \mathrm{E}$, false discovery rate $(\mathrm{FDR})>2.5 \mathrm{E}$ )

\begin{tabular}{|c|c|c|c|c|}
\hline G0 biological process & Expected & Fold enrichment & Raw p value & FDR \\
\hline Skeletal muscle contraction & 0.2 & 15.09 & $2.17 \mathrm{E}-03$ & 4.97E-02 \\
\hline Negative regulation of calcium ion transmembrane transporter activity & 0.2 & 15.09 & 2.17E-03 & 4.97E-02 \\
\hline Branch elongation of an epithelium & 0.2 & 15.09 & 2.17E-03 & 4.97E-02 \\
\hline Gland morphogenesis & 0.85 & 9.39 & $7.56 \mathrm{E}-06$ & $5.80 \mathrm{E}-04$ \\
\hline Positive regulation of endothelial cell migration & 0.74 & 8.13 & $2.12 \mathrm{E}-04$ & $8.11 \mathrm{E}-03$ \\
\hline Intrinsic apoptotic signaling pathway in response to DNA damage & 0.77 & 7.83 & $2.53 \mathrm{E}-04$ & $9.41 \mathrm{E}-03$ \\
\hline Organ growth & 0.91 & 7.7 & $8.40 \mathrm{E}-05$ & $3.86 \mathrm{E}-03$ \\
\hline Cellular response to amino acid stimulus & 0.65 & 7.65 & 9.15E-04 & $2.62 \mathrm{E}-02$ \\
\hline Lung alveolus development & 0.65 & 7.65 & $9.15 \mathrm{E}-04$ & 2.61E-02 \\
\hline Regulation of morphogenesis of a branching structure & 0.65 & 7.65 & $9.15 \mathrm{E}-04$ & $2.60 \mathrm{E}-02$ \\
\hline Cellular response to metal ion & 1.02 & 5.87 & $9.71 \mathrm{E}-04$ & $2.75 \mathrm{E}-02$ \\
\hline Myeloid cell homeostasis & 1.22 & 4.91 & $2.20 \mathrm{E}-03$ & 4.96E-02 \\
\hline Ossification & 2.33 & 4.72 & $5.06 \mathrm{E}-05$ & 2.64E-03 \\
\hline Extracellular matrix organization & 2.39 & 4.61 & $6.17 \mathrm{E}-05$ & 3.07E-03 \\
\hline Glial cell differentiation & 1.65 & 4.25 & $2.05 \mathrm{E}-03$ & 4.74E-02 \\
\hline Regulation of T-cell activation & 2.36 & 4.24 & $2.46 \mathrm{E}-04$ & $9.22 \mathrm{E}-03$ \\
\hline
\end{tabular}

cle differentiation genes. As a result, we obtained 35 calciumrelated genes in muscle differentiation and compared using Heatmap visualization to examine their expression patterns in each analysis (Figure 2). The expression patterns of calciumrelated genes were gradually up- or down-regulated during 3 days of myotube formation (Figure 2).

Evolutionary analysis and string analysis of calciumrelated differentially expressed genes

To investigate the evolutionary relationships of calcium-related candidate DEGs, we extracted and compared amino acid sequences from eight species in vertebrata (cow, human, duck, mouse, rat, dog, cat, and chicken) regarding two candidate genes, myosin light chain 1 (MYL1) and caveolin 3 (CAV3). When we conducted multiple alignment with MYL1 and CAV3, EFh_PEF super family domain in MYL1 and Caveolin domain in $C A V 3$ showed higher identity (Figure 3, solid box). Therefore, we suggest that calcium-related domains were highly conserved in the candidate genes and these domains would be interactive with calcium ions during muscle differentiation. In addition, we conducted string analysis among seven calcium related candidate genes (Figure 4). As a result, it was confirmed that four genes (MYL1, MYL3, troponin T2, cardiac type [TNNT2], troponin C1, slow skeletal and cardiac type [TNNC1]) interacted with each other.

\section{Validation of calcium-related transcript expression in QM7 cells during myotube differentiation}

To validate calcium-related transcript expression in muscle differentiation, we selected and analyzed seven genes which were gradually increased according to days of myotube differentiation periods. Similar to RNA sequencing data, the expression patterns of calcium-related transcripts were gradually and highly increased depending on days of myotube

Table 2. Biological process gene ontology (GO) terms of calcium-related differentially expressed genes (DEGs) during myotube differentiation in QM7 cells

\begin{tabular}{|c|c|c|}
\hline GO term & Gene count & Gene symbol \\
\hline Calcium channel complex & 10 & CASQ2, CAV3, CACNA1S, CACNA2D1, CACNA1B, CACNA1D, ANXA5, PDE4B, CACNG3, CACNB4 \\
\hline Calcium channel activity & 8 & CAV3, CACNA1S, CACNA1B, CACNA1D, RASA3, TRPV1, SLC24A2, CACNB4 \\
\hline Calcium mediated signaling & 15 & $\begin{array}{l}\text { CASQ2, PLN, ATP2A2, CLIC2, HDAC4, CACNA1D, RCAN3, TOX3, SLC9A1, LRRK2, KDR, ATP1B1, TPCN3, TRPV1, } \\
\text { GSTO1 }\end{array}$ \\
\hline Calcium ion binding & 16 & $\begin{array}{l}\text { MYL1, MYL3, TNNC1, CHIA, NELL2, DAG1, CAPN1, CDH2, VLDLR, CANX, CIB1, MYL9, ANXA2, EFHD1, SPTAN1, } \\
\text { FBLN5 }\end{array}$ \\
\hline Calcium ion import & 11 & CACNA2D1, ATP2A2, WNT5B, FKBP1A, CACNA1D, RASA3, CHERP, ANXA2, VDAC2, TPCN3, TRPV1 \\
\hline Calcium ion homeostasis & 29 & $\begin{array}{l}\text { CASQ2, PLN, CAV3, GRIA1, BOK, SLC8A3, SMAD3, ATP2A2, STIM1, ATP2B1, F2, HCRTR2, CRY2, FKBP1A, RASA3, } \\
\text { CHERP, PTH1R, VAPB, CD24, ATP1B1, GNB1, ANXA7, ATP13A2, GALR2, F2RL1, TPCN3, TRPV1, SLC24A2, CACNB4 }\end{array}$ \\
\hline Response to calcium & 22 & $\begin{array}{l}\text { CASQ2, TNNT2, CAPN3, SPARC, CPNE2, STIM1, DUSP1, ENTPD6, ANXA11, ANXA5, IL6, SEC31A, ANXA7, KCNIP2, } \\
\text { PPP2CA, CCND1, WNT5A, ALOX5AP, PPIF, RANBP1, SLC25A13, INHBB }\end{array}$ \\
\hline Calcium ion export & 2 & $S L C 8 A 3, A T P 2 B 1$ \\
\hline Detection of calcium & 3 & CASQ2, STIM1, KCNIP2 \\
\hline
\end{tabular}


(A)

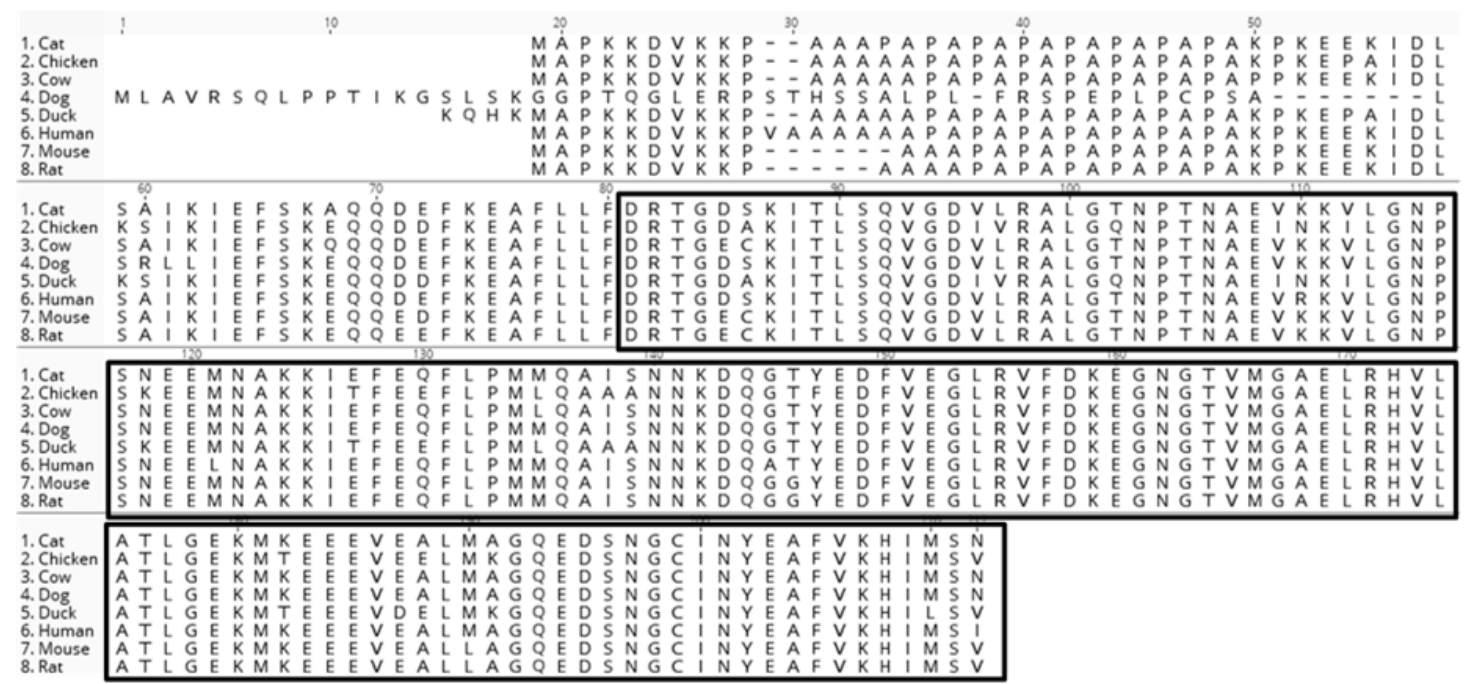

(B)

\begin{tabular}{|c|c|c|}
\hline & 10 & 80 \\
\hline $\begin{array}{l}\text { 1. Cat } \\
\text { 2. Chicken } \\
\text { 3. Cow } \\
\text { 4. Dog } \\
\text { 5. Duck } \\
\text { 6. Human } \\
\text { 7. Mouse } \\
\text { 8. Rat }\end{array}$ & 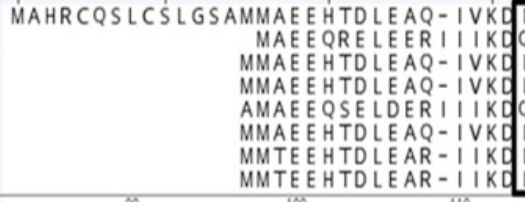 & 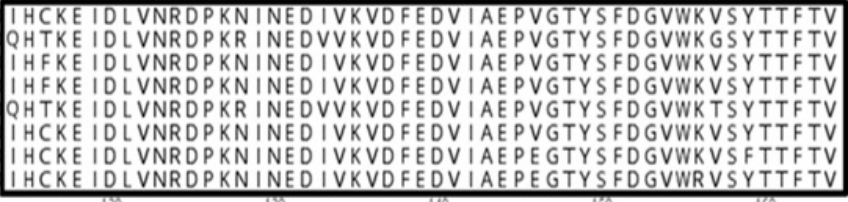 \\
\hline $\begin{array}{l}\text { 1. Cat } \\
\text { 2. Chicken } \\
\text { 3. Cow } \\
\text { 4. Dog } \\
\text { 5. Duck } \\
\text { 6. Human } \\
\text { 7. Mouse } \\
\text { 8. Rat }\end{array}$ & 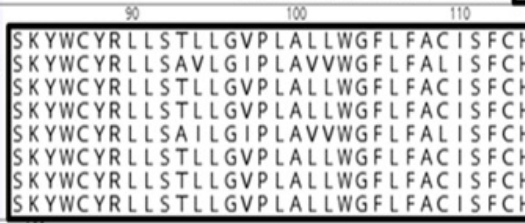 & 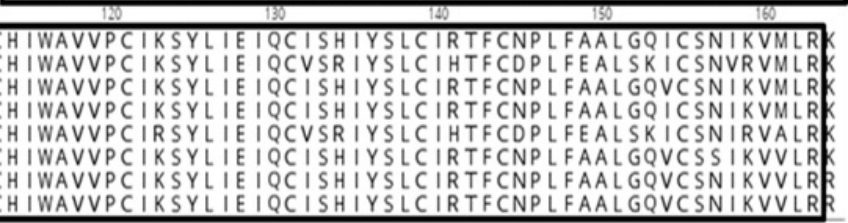 \\
\hline $\begin{array}{l}\text { 1. Cat } \\
\text { 2. Chicken } \\
\text { 3. Cow } \\
\text { 4. Dog } \\
\text { 5. Duck } \\
\text { 6. Human } \\
\text { 7. Mouse } \\
\text { 8. Rat }\end{array}$ & $\begin{array}{l}E V \\
E T \\
E V \\
E V \\
E T \\
E V \\
E G \\
E G\end{array}$ & \\
\hline
\end{tabular}

Figure 3. Comparative alignments of amino acid sequences of MYL1 and CAV3 gene which were highly expressed in myotube differentiation process. EFh_PEF super family domain of MYL1 gene (A) and Caveolin domain of CAV3 gene (B) was highly conserved in various species. The amino acid sequences were aligned by the MUSCLE method in GENEIOUS program. Each conserved domain was marked by solid box. MYL1, myosin light chain 1; CAV3, caveolin 3.

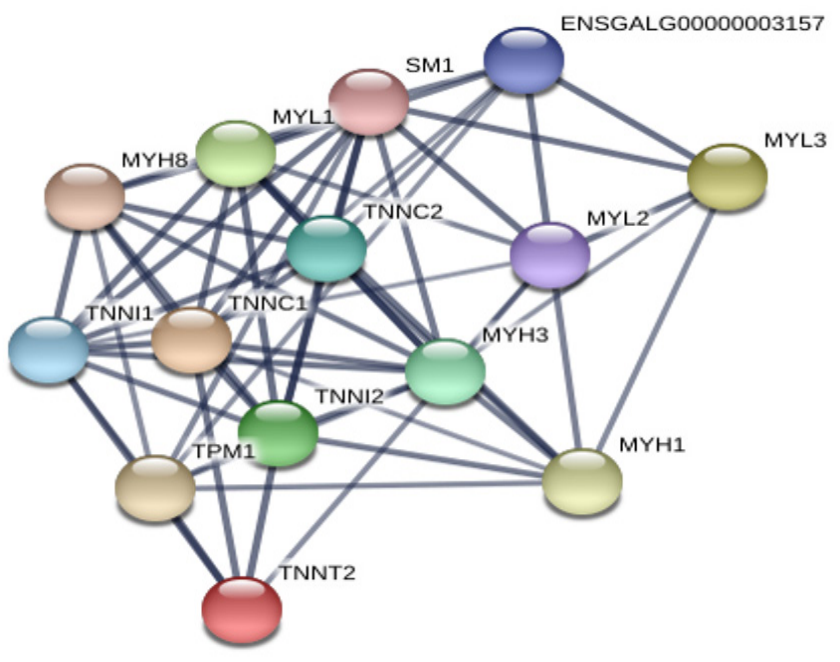

Figure 4. String analysis of calcium-related candidate genes processed by RNAsequencing data. Protein-protein interactions were analyzed with the STRING software. In the network, proteins were represented as nodes. differentiation (Figure 5). The expression level of MYL3, TNNC1, and phospholamban (PLN) transcripts were higher ( $<20$ folds) than those of undifferentiated QM7 cells while the transcription levels of MYL1, CAV3, calsequestrin 2 (CASQ2) were dramatically increased ranging from 90 to 200 fold during muscle differentiation process (Figure 5). These results may indicate that calcium-related genes such as TNNC1, MYL1, MYL3, PLN, CAV3, CASQ2, and TNNT2 play a critical role in muscle differentiation in QM7 cells.

\section{DISCUSSION}

Cell proliferation, differentiation and cell death are commonly dependent on many signaling pathways controlled by alterations of intracellular calcium [14]. In the previous study, calcium ions were utilized for regulatory molecule and signaling molecule in muscle [10]. It is well-known that calcium 

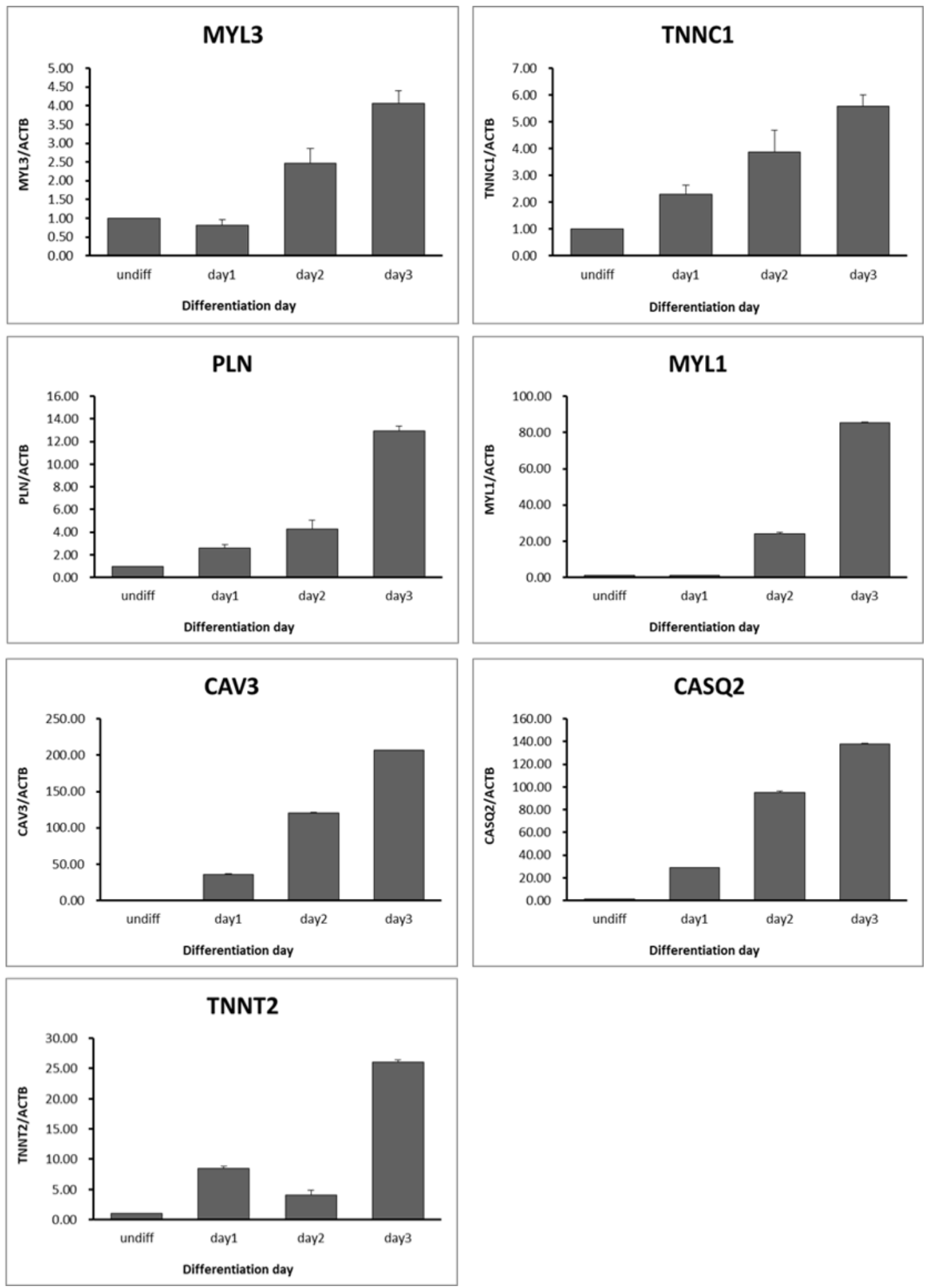

Figure 5. Expression patterns of calcium-related genes during myotube differentiation. Relative expression levels of candidate genes were analyzed by quantitative reverse-transcription polymerase chain reaction (qRT-PCR). $(n=3, p<0.005)$. Quantitative analysis was performed using the $2^{-\Delta \Delta c t}$ method by normalization with beta-actin gene.

plays a role in muscle contraction-relaxation and mechanism of muscle contraction [11]. In addition, many studies reported the biological functions of calcium ions are not only for mus- cle contraction but also for regulation of energy metabolism through ATP supply [15].

Although the calcium ion plays an important role in muscles, 
the biological effects of calcium during muscle differentiation have not been well studied. Previous researches indicated that intracellular calcium acted an important role in muscle-specific gene expression, and calcium dependent transcriptional pathway was closely related to skeletal muscle hypertrophic growth [16]. Other studies showed the results of calcium effects on muscle differentiation in frog and rat [17]. In these studies, one of the calcium binding proteins, parvalbumin was identified in myogenesis of frog and rat and detected particularly during the differentiation process of myotomal muscle [18]. Heizmann and Strehler [19] also presented experimental results suggesting the physiological role of parvalbumin in chicken muscle cells. In myotube development of chick embryos, the calcium regulatory system is modulated during myofibiliogenesis and expression level of avian calsequestrin homolog containing calcium storage capacity increased 10-fold before myoblast fusion [20]. These results suggest a biologically significant effect of calcium on muscle differentiation.

In this study, we identified calcium-related genes which presented the up-regulated patterns during myotube differentiation and investigated the binding structure with the highly conserved domains between various species (Figure 3). The expression of MYL1 gene increased in the myotube differentiation-dependent manner [21] and was significantly detected at an early stage of fast twitch fiber differentiation in zebrafish embryo [22]. The higher similarity of EFh_PEF super family domain sequences in MYL1 and CAV3 gene might be evolutionary evidence of muscle formation in vertebrates. When two or more genes have been derived from a common ancestor, these genes are called orthologous genes and contain a similar domain. These orthologous genes may perform a similar function and so could be evolutionary evidence [23]. Calsequestrin (CASQ) is a main calcium binding protein located within the lumen of sarcoplasmic reticulum [24]. A recent report showed that CASQ regulated a RyR channel are closely related to development of skeletal muscle [25]. Particularly, Schwartz and Kay [18] reported that the expression level of CASQ2 considerably increases immediately before myoblast fusion. The regulatory function of TNNC1 (also known as troponin-C type 1) which is one of calcium binding proteins was reported as calcium ion release from the sarcoplasmic reticulum bind troponin subunits ( $\mathrm{TnC}$ ). After binding of calcium ion with troponin subunits, muscle initiates the contraction. Similarly, this modulatory machinery was also observed during myoblast fusion in quail [26]. Collectively, the results presented indicate that calcium-related candidate genes play important roles in avian muscle differentiation.

\section{CONFLICT OF INTEREST}

We certify that there is no conflict of interest with any financial organization regarding the material discussed in the manu- script. Kang KS is an employee of Bio Division, Medikinetics, Inc.

\section{ACKNOWLEDGMENTS}

This work was carried out with the support of "Cooperative Research Program for Agriculture Science and Technology Development (Project No. PJ01334801)" Rural Development Administration, Republic of Korea.

\section{REFERENCES}

1. Van Kaam JB, Groenen MA, Bovenhuis H, et al. Whole genome scan in chickens for quantitative trait loci affecting growth and feed efficiency. Poult Sci 1999;78:15-23.

2. Yaffe D. Retention of differentiation potentialities during prolonged cultivation of myogenic cells. Proc Natl Acad Sci USA 1968;61;477-83.

3. Arnold HH, Winter B. Muscle differentiation: more complexity to the network of myogenic regulators. Curr Opin Genet Dev 1998;8:539-44.

4. Kim SW, Lee JH, Park BC, Park TS. Myotube differentiation in clustered regularly interspaced short palindromic repeat/ Cas9-mediated MyoD knockout quail myoblast cells. AsianAustralas J Anim Sci 2017;30:1029-36.

5. Kim SW, Lee JH, Park TS. Functional analysis of SH3 domain containing ring finger 2 during the myogenic differentiation of quail myoblast cells. Asian-Australas J Anim Sci 2017;30: 1183-9.

6. Kerst B, Mennerich D, Schuelke M. et al. Heterozygous myogenic factor 6 mutation associated with myopathy and severe course of Becker muscular dystrophy. Neuromuscul Disord 2000;10:572-7.

7. Miner JH, Wold B. Herculin, a fourth member of the MyoD family of myogenic regulatory genes. Proc Natl Acad Sci USA 1990;87:1089-93.

8. Buckingham M, Rigby PW. Gene regulatory networks and transcriptional mechanisms that control myogenesis. Dev Cell 2014:28:225-38.

9. Lansdown AB. Calcium: a potential central regulator in wound healing in the skin. Wound Repair Regen 2002;10:271-85.

10. Tharin S, Hamel PA, Conway EM, et al. Regulation of calcium binding proteins calreticulin and calsequestrin during differentiation in the myogenic cell line L6. J Cell Physiol 1996;166: 547-60.

11. Ledoux J, Werner ME, Brayden J, et al. Calcium-activated potassium channels and the regulation of vascular tone. Physiology 2006;21:69-78.

12. Ohtsuki I, Maruyama K, Ebashi S. Regulatory and cytoskeletal proteins of vertebrate skeletal muscle. Adv Protein Chem 1986; 38:1-67.

13. Rees DD, Frederiksen DW. Calcium regulation of porcine aortic 
myosin. J Biol Chem 1981;256:357-64.

14. Orrenius S, Zhivotovsky B, Nicotera P. Calcium: Regulation of cell death: the calcium-apoptosis link. Nat Rev Mol Cell Biol 2003;4:552-65.

15. Westerblad H, Bruton JD, Katz A. Skeletal muscle: energy metabolism, fiber types, fatigue and adaptability. Exp Cell Res 2010;316:3093-9.

16. Musarò A, McCullagh KJ, Naya FJ, et al. IGF-1 induces skeletal myocyte hypertrophy through calcineurin in association with GATA-2 and NF-ATc1. Nature 1999;400:581-5.

17. Kay BK, Shah AJ, Halstead WE. Expression of the Ca2+-binding protein, parvalbumin, during embryonic development of the frog, Xenopus laevis. J Cell Biol 1987;104:841-7.

18. Schwartz LM, Kay BK. Differential expression of the $\mathrm{Ca}^{2+}$-binding protein parvalbumin during myogenesis in Xenopus laevis. Dev Biol 1988;128:441-52.

19. Heizmann CW, Strehler EE. Chicken parvalbumin. Comparison with parvalbumin-like protein and three other components ( $\mathrm{Mr}=8,000$ to 13,000). J Biol Chem 1979;254:4296-303.

20. Froemming GR, Ohlendieck K. Oligomerisation of $\mathrm{Ca}^{2+}$-regulatory membrane components involved in the excitationcontraction-relaxation cycle during postnatal development of rabbit skeletal muscle. Biochim Biophys Acta Protein Structure and Molecular Enzymology 1998;1387:226-38.

21. Billeter R, Quitschke W, Paterson BM. Approximately 1 kilobase of sequence 5 to the two myosin light-chain $1 \mathrm{f} / 3 \mathrm{f}$ gene cap sites is sufficient for differentiation-dependent expression. Mol Cell Biol 1988;8:1361-5.

22. Burguière AC, Nord H, von Hofsten J. Alkali-like myosin light chain-1 (myl1) is an early marker for differentiating fast muscle cells in zebrafish. Dev Dyn 2011;240:1856-63.

23. Bitard-Feildel T, Kemena C, Greenwood JM, Bornberg-Bauer E. Domain similarity based orthology detection. BMC Bioinformatics 2015; 16:154.

24. Parton RG, Way M, Zorzi N, et al. Caveolin-3 associates with developing T-tubules during muscle differentiation. J Cell Biol 1997;136:137-54.

25. Fliegel L, Ohnishi M, Carpenter MR, et al. Amino acid sequence of rabbit fast-twitch skeletal muscle calsequestrin deduced from cDNA and peptide sequencing. Proc Natl Acad Sci USA 1987;84:1167-71.

26. Devlin RB, Emerson Jr, CP. Coordinate regulation of contractile protein synthesis during myoblast differentiation. Cell 1978;13:599-611. 